\title{
Delian Metamorphoses
}

\author{
Norbert Hungerbühler and Michael Nüsken
}

Norbert Hungerbühler wurde 1964 in Flawil (SG) geboren. Nach seiner Promotion 1994 an der ETH Zürich folgten mehrere Auslandsaufenthalte in Deutschland und den USA. Seit Herbst 2000 ist er Professor an der Universität Fribourg. Sein hauptsächliches mathematisches Interesse gilt nichtlinearen partiellen Differentialgleichungen.

Michael Nüsken wurde 1967 in Lüdenscheid (D) geboren. Nach seiner Promotion 1998 an der Universität Konstanz war er an der Universität Paderborn tätig und ist seit Dezember 2004 am b-it (Bonn-Aachen International Center for Information Technology). Seine Hauptinteressen reichen von der Algebra über die Computeralgebra bis in die Kryptographie.

Ernst Specker (2002, Aufgabe 1184) asked:

Nach einem Orakelspruch sollte die Pest in Griechenland dann zu Ende gehen, wenn der würfelförmige Altar im Apollonheiligtum auf Delos verdoppelt werde. Nach traditioneller Interpretation verlangt der Spruch die (unmögliche) Konstruktion der Kante des neuen Altars mit Zirkel und Lineal. Bei Ausgrabungen auf Delos soll nun aber ein Granitquader, dessen Kanten sich wie $2: 1: 1$ verhalten, gefunden worden sein. Damit drängt sich eine

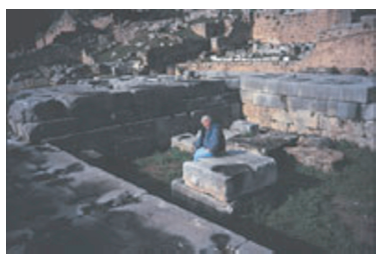

Fig. 0.1 Granite cuboid found on Delos

Die Würfelverdoppelung gehört zu den klassischen Problemen der antiken Geometrie. Die Unmöglichkeit einer konstruktiven Lösung mit Zirkel und Lineal wurde erst im 19. Jahrhundert gezeigt. Allerdings existieren Lösungen mit anderen Hilfsmitteln, wie etwa durch ,paper folding“. In diesem Artikel wird eine Lösung vorgeschlagen, bei welcher ein $2 \times 1 \times 1$-Quader in sieben polyedrische Teile zerlegt wird, die zu einem Würfel zusammengesetzt werden können. Es wird auch gezeigt, dass die Lösung durch eine Doppelkachelung des Raumes erzeugt werden kann. 
neue Deutung des Orakelspruchs auf: Ein Quader vom Typus $2: 1: 1$ ist so (in endlich viele Polyeder) zu zerlegen, dass sich aus den Teilen ein Würfel bilden lässt. Gibt es eine solche Zerlegung?

Wenn ja, gebe man eine

- leicht zu realisierende Zerlegung für Steinmetze und Puzzlefreunde an.

- Zerlegung mit einer (möglichst) kleinen Anzahl von Stücken an. ${ }^{1}$

The question of equidecomposable polyhedra is a very old one: Already in ancient Greek mathematics, the areas of triangles and parallelograms had been determined by dissection of the figures. In principle already the ancient Greek mathematicians could have proved, using only Euclid's methods (and the Archimedean axiom to be precise), that any two polygons (plane polyhedra) have the same area if and only if they can be cut into the same set of finitely many polygonal pieces. We will come back to this remarkable fact later on. Specker's challenge is about the corresponding problem in space. In 1844, in two letters to Gerling, Gauß was wondering that, in order to prove that two mirror symmetric tetrahedra have the same volume, it seemed necessary to use the method of exhaustion. After Gerling found a satisfactory answer to this question, Gauß pointed out to Gerling that exhaustion, which means dissection into infinitely many pieces, is also needed for other theorems of stereometry like Euclid XII.5, where it is shown that the volumes of two pyramids of the same height have the same ratio as the area of their bases. Hilbert, in his famous talk at the 1900 International Congress of Mathematics in Paris, took up this phenomenon and asked in his third problem to find two tetrahedra of same base and height that are not equidecomposable. Only a few months later, Max Dehn (1900) answered the question by defining a quantity which remains invariant under dissection, yet has different values for a cube and a regular tetrahedron. More details follow but first we turn back to Specker's original problem.

\section{An elementary approach}

A person who is inexperienced in dissection problems risks taking the bait and trying to dissect the altar, i.e., the $2 \times 1 \times 1$-cuboid, by cuts that are parallel to its axes, and then reassembling the pieces to form the cube. How could this be achieved? First we cut a $1 \times 1 \times 1$-cube, then three slices $1 \times 1 \times(\sqrt[3]{2}-1)$. This yields a cube with three pasted slices such that three edges already have the correct length, see Fig. 1.1. To complete the cube we would still need three beams $1 \times(\sqrt[3]{2}-1) \times(\sqrt[3]{2}-1)$ and a cube with side-length $\sqrt[3]{2}-1$.

\footnotetext{
${ }^{1}$ According to an oracle the pest in Greece would come to an end when the cubic altar in Apollo's sanctuary on Delos was doubled. In the traditional interpretation the verdict asked for the (impossible) construction of the edge of the new altar with ruler and compass. From excavations on Delos it rumoured that a granite cuboid was found whose edge proportions are 2:1:1. This leads to a new interpretation of the oracle's spell: A cuboid of type $2: 1: 1$ has to be dissected (into finitely many polyhedra) such that the pieces can form a cube. Does such a dissection exist?

If so give a

- easily realizable dissection for stonemasons and puzzle friends.

- dissection with as few pieces as possible.
} 
The leftover of the altar is a slightly thinner piece of size $1 \times 1 \times(4-3 \sqrt[3]{2})$. From this piece we cut three beams of height $\sqrt[3]{2}-1$ and then four slices $1 \times(4 \sqrt[3]{2}-5) \times(4-3 \sqrt[3]{2})$. From the fourth of these slices we cut three rods with square cross section. These pieces can be put together to obtain the three needed beams $1 \times(\sqrt[3]{2}-1) \times(\sqrt[3]{2}-1)$. There remains a beam $1 \times(4-3 \sqrt[3]{2}) \times(24-19 \sqrt[3]{2})$ and a rod $1 \times(19-15 \sqrt[3]{2}) \times(5-4 \sqrt[3]{2})$ which have to be dissected into the missing cube of side-length $\sqrt[3]{2}-1$. And so on...
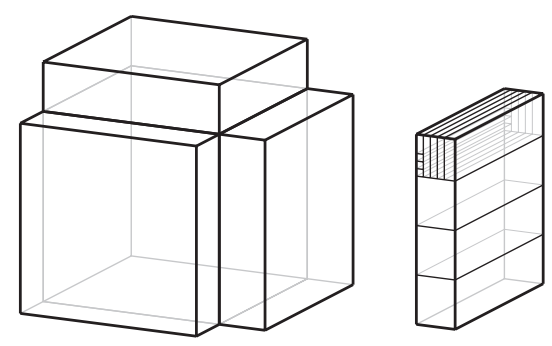

Fig. $1.1 \sqrt[3]{2}-1 \approx 0.2599,4-3 \sqrt[3]{2} \approx 0.2202$

At this point, the reader will suspect that this method will not terminate. Indeed, all sidelengths that occur up to now are of the form $a+b \sqrt[3]{2}$ with integers $a$ and $b$. Since $\sqrt[3]{2}$ is irrational, we may presume that no such finite dissection of altar and cube exists. Of course, seemingly unmotivated cuts may destroy the side-length pattern observed so far and this argument falls apart. Anyhow, the reader might still be convinced that no finite common dissection of altar and cube into cuboids exists. However, cuts in other directions could still lead to the desired result. We just did not try it yet because it seems much more difficult to visualize. So we first go back into two dimensions and consider the analogous problem of dissecting a square into a rectangle. Here the answer turns out to be surprisingly simple.
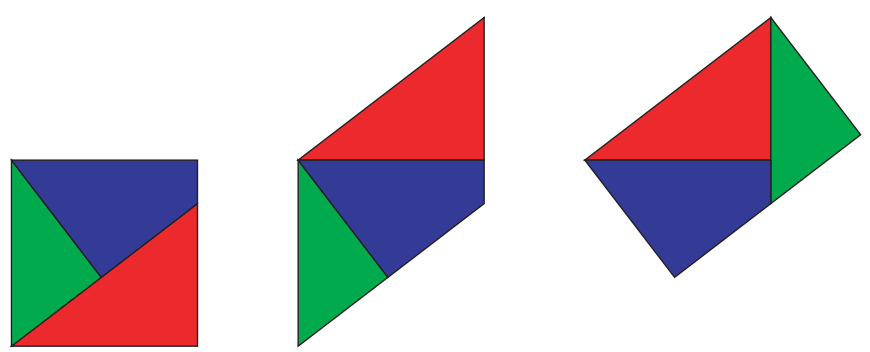

Fig. 1.2 Dissection of a $\sqrt[3]{2}$-square into a $\sqrt[3]{4} \times 1$-rectangle

Starting with a rectangle we cut off a triangle, like in Fig. 1.2, and move it to the opposite side which results in a parallelogram. From this figure we come back to a different rectangle by a similar operation: the triangle on the left is shifted to the right. These two steps lead from one rectangle to another rectangle. The method is quite flexible, we can choose the height of the new rectangle by adjusting the slope of the first cut. Even if the slope is getting too steep, as in Fig. 1.3, we can still cyclically continue to cut and arrive at 
a rectangle whose height is the distance of the top left corner of the original rectangle to the line given by the first cut. In general, there will be more than just three pieces, but the method allows us to dissect any rectangle into a rectangle of equal area and height 1 . This generalizes easily to the following famous theorem.
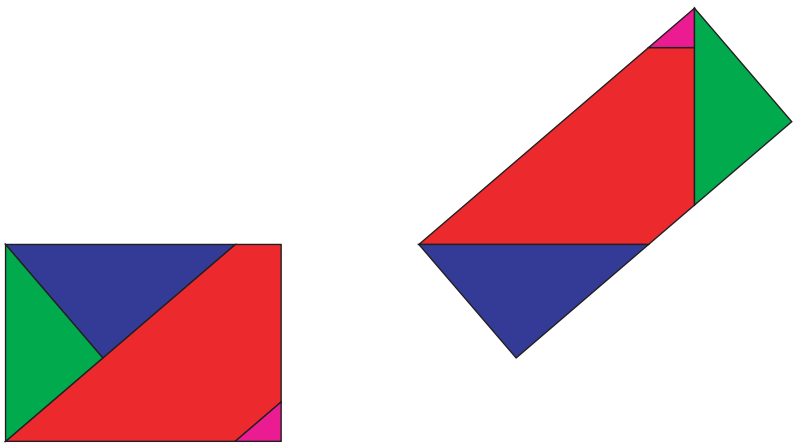

Fig. 1.3 Dissection of a rectangle into a narrow one

Theorem 1.1 (Wallace (1831); Lowry (1814); Bolyai (1832); Gerwien (1833)). Two polygons are equidecomposable if and only if they have the same area.

Two polyhedra $P^{(1)}$ and $P^{(2)}$ are called equidecomposable or scissors congruent if both allow a finite, almost disjoint decomposition $P^{(i)}=\bigcup_{j=1}^{n} P_{j}^{(i)}$ such that $P_{j}^{(1)}$ and $P_{j}^{(2)}$ are congruent polyhedra. ("Almost disjoint" means that the interiors of $P_{j_{1}}^{(i)}$ and $P_{j_{2}}^{(i)}$ are disjoint for all $i, j_{1}, j_{2}$.)

It is trivial that equidecomposable polyhedra have equal volume. The surprising inverse implication in two dimensions stated in the theorem was proved by Bolyai (1832) and Gerwien (1833) independently. In fact, it is quite likely that Wallace had proved the theorem already in 1808. The problem he posed was explicitly solved by Lowry (1814). Wallace's own extended solution was published in Wallace (1831). The proof is amazingly simple: because equidecomposability is an equivalence relation it suffices to dissect any polygon into a rectangle of height 1 . Since every polygon possesses a triangulation, and hence can be dissected into triangles, and since, as we have seen, all rectangles of equal area are equidecomposable, we are left with the problem of showing that every triangle can be dissected into a rectangle. This is easy, as indicated in Fig. 1.4. Theorem 1.1 remains valid in two-dimensional hyperbolic and elliptic geometry.

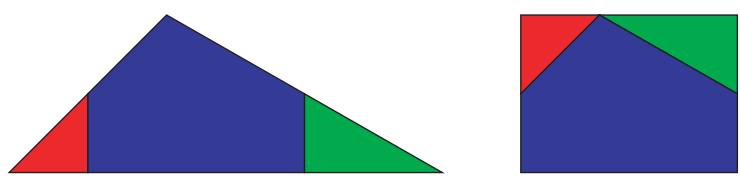

Fig. 1.4 Dissection of a triangle into a rectangle 
After the preparatory work in two dimensions the transfer to three dimensions is easily accomplished. A dissection of two rectangles yields a dissection of two cuboids if we just apply the plane pattern to a face of a cuboid and cut it like a pie orthogonal to the face. By performing this operation twice with respect to two adjacent faces of a given cuboid $C$ we obtain a common dissection of three cuboids, and we may consider the given cuboid $C$ as an intermediate step between the two resulting cuboids. In Fig. 1.6 a $\sqrt[3]{4} \times \sqrt[3]{2} \times 1$-cuboid is dissected by applying two plane patterns: the front is dissected using the pattern from Fig. 1.2, and to the top the pattern on the left of Fig. 1.5 is applied. Thus, the resulting pieces can be reassembled to form both the cube and the altar! The resulting description for stone masons and puzzle friends might therefore read as follows.
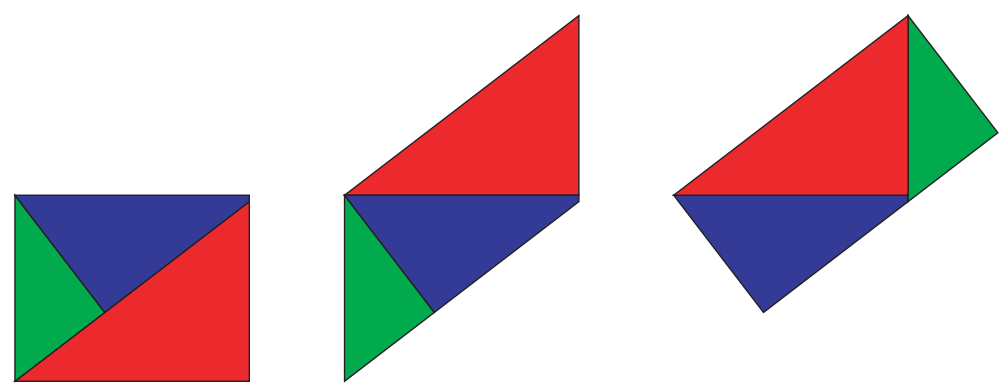

Fig. 1.5 Dissection of a $\sqrt[3]{4} \times \sqrt[3]{2}$-rectangle into a $2 \times 1$-rectangle

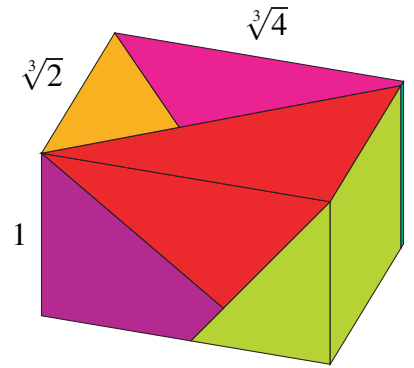

Fig. 1.6 Intermediate $\sqrt[3]{4} \times \sqrt[3]{2} \times 1$-cuboid

Answer 1.2. Apply the dissection on the right of Fig. 1.5 to the altar, cut accordingly and reassemble the pieces as indicated on the left of Fig. 1.5. Continue by applying the dissection on the right of Fig. 1.2 as in the front of Fig. 1.6, cut again and reassemble the pieces according to the dissection on the left of Fig. 1.2. Done!

What we found is a solution with no more than nine pieces which relies on the common dissection of two rectangles into three pieces.

Let us take a closer look at the dissection we found. A direct inspection reveals immediately that one of the nine pieces is empty. Indeed, Fig. 1.7 shows that the left prism induced by the top dissection does not meet the right prism induced by the front dissection. 


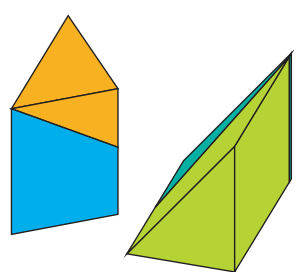

Fig. 1.7 Disjoint prisms

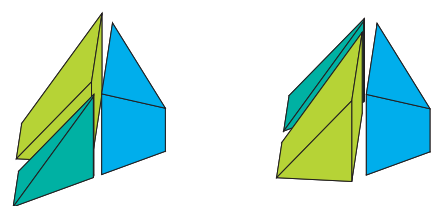

Fig. 1.8 The critical pieces in the 8-piece solution.

At this point, the second author produced several Maple animations. In one of them, the pieces move from their position in the altar straight to their position in the cube. Of course the pieces interpenetrate on their way from one position to the other. Nevertheless, we can learn something: it looks as if two of the remaining eight pieces move simultaneously and could therefore be glued together to form one single piece. The two pieces concerned are the ones that build the right and the left face of the cuboid in Fig. 1.6. If we could glue these two slices we would have a dissection with only seven pieces. We could, however, in that case no longer build the intermediate $\sqrt[3]{4} \times \sqrt[3]{2} \times 1$-cuboid, but that would not hurt. Another observation originated from the Maple animations, namely that almost all faces of the eight pieces are once on the inside and once on the outside of the altar or the cube. The only faces that are twice inside are precisely the faces we just intended to glue together. A closer inspection unfortunately showed that the two pieces we have in mind actually do slide relative to each other but only a very small distance that corresponds exactly to their difference in size, i.e., the length of the shortest edge in the quadrilateral in Fig. 1.5. (There is a quadrilateral!) Being so close to a solution with only seven pieces it seemed reasonable to ask if there might be, nonetheless, a solution with seven pieces only. At this point another piece comes into play, namely the piece which is located once on one side and once on the other side of the gliding piece, and which compensates exactly the small distance by which the two original pieces move relative to each other, see Fig. 1.8. Let us try to cut off a slice of one piece and glue it to the other one. Then the displacement should no longer be necessary and we could glue the two pieces. And indeed, this plan works!

Theorem 1.3. There exists a common dissection of altar and cube with seven pieces.

Fig. 1.9 shows how the seven pieces build up the altar and the cube, respectively. The web page Nüsken (2003) offers an animation illustrating the solution.

The solution in Fig. 1.9 reveals a wonderful and surprising property, namely:

Observation 1.4. If we consider the faces of the seven pieces, once if they form the cube and once if they form the altar, then each face is one time on the inside and the other time on the outside. None of the faces is in both configurations hidden inside or in both configurations visible on the surface.

Note further that the seven pieces in our solution only need to be displaced by translations, provided the cube and the altar have a suitable orientation relative to each other. In this 

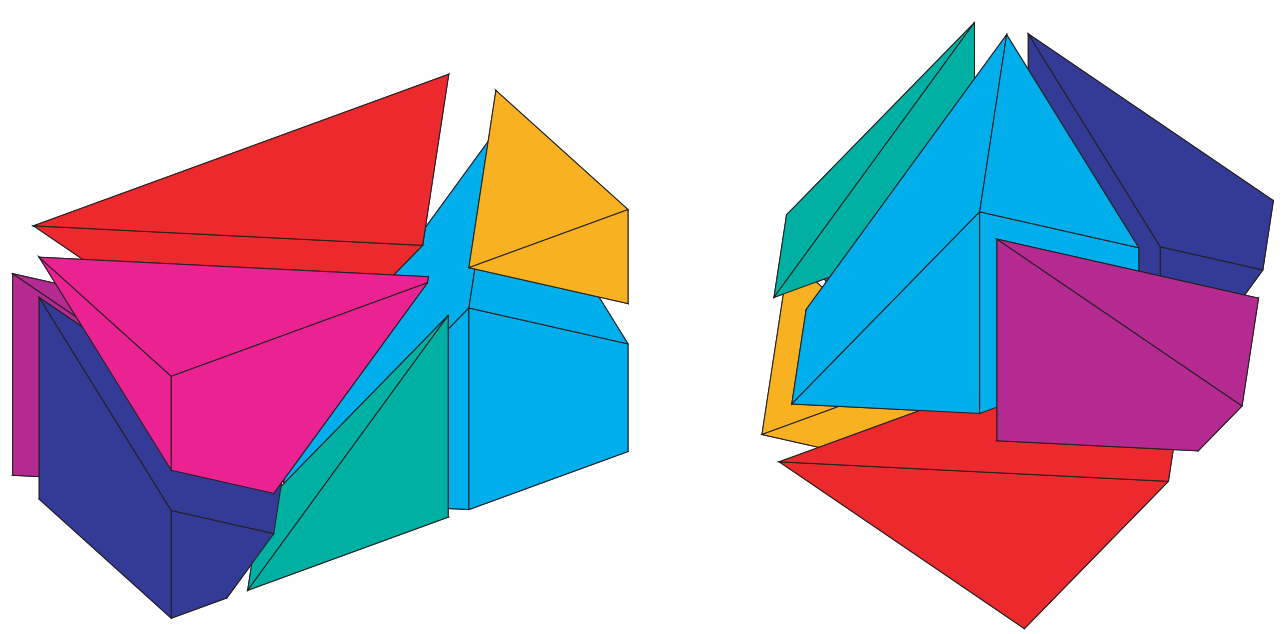

Fig. 1.9 Dissection of altar and cube with seven pieces

orientation, cube and altar have no parallel faces. It is therefore impossible that a face of one of the pieces is in both cases on the outside. Since we can move the pieces from one body to the other without rotating them, the dissection is called translational.

Hadwiger \& Glur (1951) have shown that two arbitrary plane polygons having equal area are equidecomposable even if only translations and point symmetries are allowed for the conversion. After the considerations we made earlier for plane polygons, we can almost prove this result: in addition, we would only need to show that one can turn a triangle or a rectangle by using only the means allowed. This is actually impossible using translations alone, as the following characterization shows:

Theorem 1.5 (Hadwiger \& Glur (1951)). Two polygons $P$ and $Q$ of equal area are translation equidecomposable if and only if the functions $\Phi_{P}$ and $\Phi_{Q}$ agree.

Here, for a unit vector $v$ in the plane, $\Phi_{P}(v)$ is defined to be the sum of the oriented lengths of those edges of $P$ that are perpendicular to $v$. In this sum the length counts positive if $v$ is an outer normal vector and negative in the opposite case.

For a rectangle $R$ the function $\Phi_{R}$ vanishes identically, but for a triangle this is not the case. Therefore, a rectangle and a triangle cannot be translationally equidecomposable, no matter how they are oriented.

\section{Dissection in space and the Dehn invariant}

Let us come back to Hilbert's third problem:

[...] specifying two tetrahedra of equal bases and equal altitudes which can in no way be split up into congruent tetrahedra, and which cannot be combined with congruent tetrahedra to form two polyhedra which themselves could be split up into congruent tetrahedra. 
Hilbert does not only consider the notion of equidecomposability but also polyhedra that are equivalent by completion. Two polyhedra that are equidecomposable are trivially equivalent by completion. Surprisingly, the inverse implication is also true: for two dimensions, this follows from Theorem 1.1. In higher dimensions, the issue becomes more subtle: Sydler (1943/44) has shown the corresponding result in three dimensions and Hadwiger (1952a) generalized it to arbitrary dimensions (and even arbitrary transformation groups $G$ which contain all translations). A complete proof is also provided in Hadwiger (1957, 2.2.1).

Max Dehn (1900) solved Hilbert's third problem shortly after the congress. He was able to show that a cube and a tetrahedron of the same volume are not equidecomposable. The proof is meanwhile so simple that we can present its main features here: the decisive tool is the Dehn invariant. Its crucial property is, as its name indicates, to be invariant under dissection of a polyhedron into several pieces. In other words: two equidecomposable polyhedra must have the same Dehn invariant. In order to compute the value of the Dehn invariant of a given polyhedron $P$, one has to determine the length $l_{e} \in \mathbb{R}_{\geq 0}$ of every edge $e$ of $P$ and the spherical angle $\alpha_{e} \in \mathbb{R} / \pi \mathbb{Z}$ modulo $\pi$ between the two faces adjacent to $e$. In modern notation, the Dehn invariant takes values in the tensor product $\mathbb{R} \otimes_{\mathbb{Z}} \mathbb{R} / \pi \mathbb{Z}$ and is given by

$$
D(P):=\sum_{\substack{e \text { edge } \\ \text { of } P}} l_{e} \otimes \alpha_{e} \in \mathbb{R} \otimes_{\mathbb{Z}} \mathbb{R} / \pi \mathbb{Z} .
$$

The tensor property $l \otimes(\alpha+\beta)=l \otimes \alpha+l \otimes \beta$ reflects what happens if one cuts along an edge, dissecting the angle between the faces into two angles $\alpha$ and $\beta$. Similarly, the tensor property $(l+m) \otimes \alpha=l \otimes \alpha+m \otimes \alpha$ corresponds to cutting an edge, dissecting its length into two parts $l$ and $m$. Measuring the angles modulo $\pi$ allows one to introduce new (first only virtual) edges with face angle $\pi$. Our cube has twelve edges of length $\sqrt[3]{2}$ and all angles are $\frac{\pi}{2}$. Hence, we have $D($ Cube $)=12 \cdot\left(\sqrt[3]{2} \otimes \frac{\pi}{2}\right)=\sqrt[3]{2} \otimes 6 \pi=0$. On the other hand, a regular tetrahedron of the same volume possesses six edges of equal length $l$ and all angles are $\alpha \approx 70.5^{\circ}$ with $\cos \alpha=\frac{1}{3}$. Hence, its Dehn invariant is given by $6 l \otimes \alpha$. Now, $6 l \otimes \alpha$ vanishes if and only if $6 l=0$ or if $\alpha$ is a rational multiple of $\pi$. Dupont (2001) gives the following nice argument which shows that $\alpha / \pi$ is irrational: it suffices to verify that for every $k \in \mathbb{N}_{>0}$ the number $3^{k} \cos k \alpha$ is an integer and not a multiple of three. To see this we use the formula $\cos k \alpha=2 \cos \alpha \cdot \cos (k-1) \alpha-\cos (k-2) \alpha$. 「The assertion is obviously true for $k=1$. Suppose $k>1$. Then $3^{k} \cos k \alpha=2\left(3^{k-1} \cos (k-\right.$ $1) \alpha)-9\left(3^{k-2} \cos (k-2) \alpha\right)$ and by induction we see immediately that this expression does not vanish modulo 3.」 We conclude that

$$
D(\text { Tetrahedron }) \neq 0=D(\text { Cube })
$$

and hence the cube and the tetrahedron cannot be equidecomposable since their Dehn invariants disagree. (Moreover, they cannot be equivalent by completion as Hilbert asked.) Going back to our original task, let us determine the Dehn invariant of the altar: all its angles are $\frac{\pi}{2}$ and hence $D($ Altar $)=1 \otimes 8 \cdot \frac{\pi}{2}+2 \otimes 4 \cdot \frac{\pi}{2}=1 \otimes 8 \pi=0$. Thus, the cube and the altar have the same Dehn invariant. Surprisingly, the converse of Dehn's result is also true: if two three-dimensional polyhedra of equal volume have the same Dehn invariant they are equidecomposable. Not until 25 years after Hopf pointed out this problem to 
his student, Sydler (1965) proved this theorem. Jessen $(1968,1972)$ and Dupont \& Sah (1990) later shortened the proof considerably. In addition, Jessen (1972) was able to show that the analogous criterion holds in four dimensions for equidecomposable polytopes. In higher dimensions however, and in hyperbolic and elliptic geometry in dimension three and above, the problem remains open, Kellerhals (1999). For our original problem it follows from Sydler's theorem that the cube and the altar are indeed equidecomposable (as we know already). However, the theorem does not tell us what a common dissection looks like, let alone one with a minimum number of pieces.

Before we continue we want to reveal that the conjecture that a common dissection of altar and cube into finitely many cuboids does not exist is indeed correct. Spandaw (2002) or Hadwiger (1957, 2.2.10, Korollar I), describe this in a lucid way:

Theorem 2.1 (Hadwiger (1952c); Spandaw (2002)). Two cuboids of equal volume have a common dissection into cuboids if and only if their edges are, up to a permutation, rational multiples of each other.

Since $\sqrt[3]{2}$ is irrational, our problem is thus insoluble this way. Here again, the decisive tool is an invariant: we associate to a $a_{1} \times a_{2} \times a_{3}$ cuboid the value $S\left(a_{1} \times a_{2} \times a_{3}\right):=$ $\frac{1}{6} \sum_{\sigma \in \mathcal{S}_{3}} a_{\sigma(1)} \otimes a_{\sigma(2)} \otimes a_{\sigma(3)} \in \mathbb{R} \otimes_{\mathbb{Z}} \mathbb{R} \otimes_{\mathbb{Z}} \mathbb{R}$. This value is invariant under a permutation of the axes and under a dissection into smaller cuboids. Of course, we can easily calculate this invariant for our cube and the altar: $S($ Altar $)=2 \otimes 1 \otimes 1$ and $S($ Cube $)=\sqrt[3]{2} \otimes$ $\sqrt[3]{2} \otimes \sqrt[3]{2}$, which are obviously two different values. Observe that we may relocate integer factors but, after all, $\sqrt[3]{2}$ is irrational. Partial results concerning rectangular dissections can be found already in Dehn (1902). However, they do not cover our case, since his main interest was the relation of the pieces to the ensemble.

To the present day, only a few tetrahedra are known which are equidecomposable with a cube: there are the three one-parameter families of tetrahedra of Hill (1896) and in addition 27 isolated tetrahedra which are completely listed in Boltianksiǔ $(1978, \S 18)$. A particularly nice and elementary proof of the equidecomposability of Hill's tetrahedra to a straight prism which uses only six pieces is described in Schöbi (1985). Such a prism is according to Theorem 1.1 equidecomposable to a cuboid.

\section{Dissections and tilings}

A nice riddle asks for a dissection of the cross in Fig. 3.1, which is built from five squares, such that the pieces can be reassembled to form a square. A trial and error strategy may take

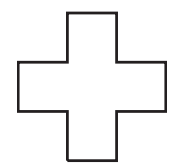

Fig. 3.1 A cross built from five squares

a while and may lead to quite a large number of pieces. However, there is an astonishing method to generate an answer. The method starts with a tiling of the plane by copies of 


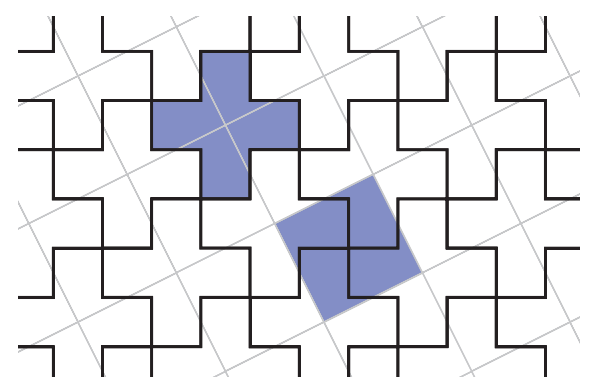

Fig. 3.2 Double tiling of the plane

the crosses. This is, of course, not possible with every polygon but the cross works just fine. Then Fig. 3.2 shows where we can find the desired square. Indeed, we find quite automatically a second tiling of the plane by the square in such a way that the periods of both tilings agree. If we cut one of the crosses along the square tiling we get precisely the common dissection of the square and the cross with only four pieces! Several of Escher's famous tilings can be interpreted in a similar manner: in "Reptiles" (Fig. 3.3) we find a tiling in which every lizard is equidecomposable to a regular hexagon.

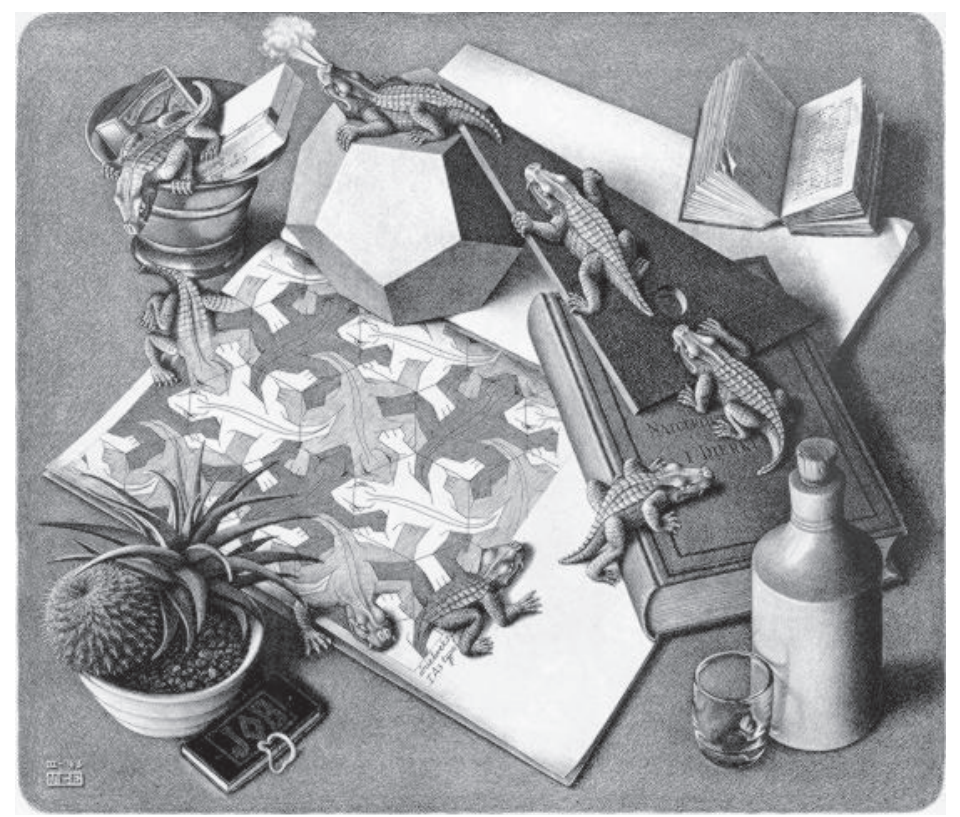

Fig. 3.3 M.C. Escher: Reptiles, lithography, 1943

Mathematically speaking a double tiling is given by a lattice $\Gamma \in \mathbb{R}^{n}$ and two fundamental domains $E$ and $F$, that is $\Gamma+E$ covers $\mathbb{R}^{n}$ almost disjointly and $\Gamma+F$ does that as well. The tiling method has led to many dissections with surprisingly few pieces. Already Mahlo 


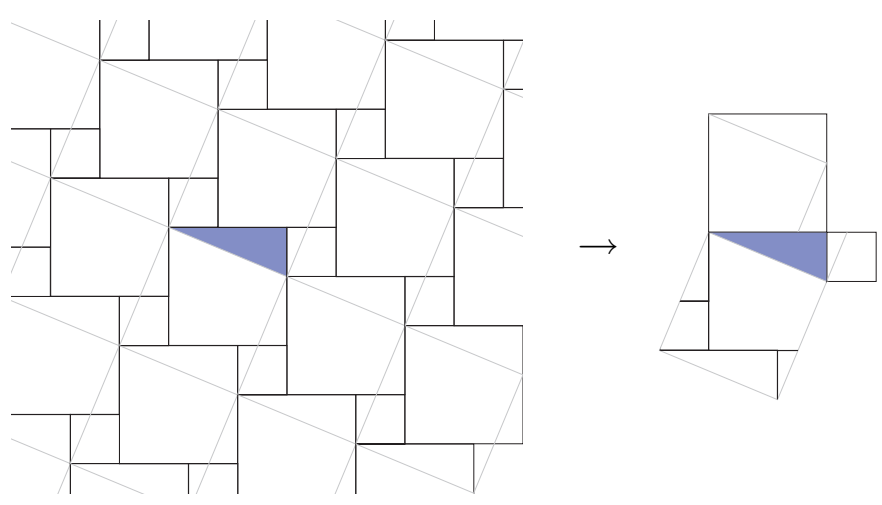

Fig. 3.4 Pythagorean metamorphosis

(1908) and MacMahon (1922) noticed that one can prove the pythagorean theorem using the double tiling in Fig. 3.4. See also Rogers (2005) for a more detailed presentation of this part of the story.

Common dissections originating from the tiling method with only parallel translations of copies of a polygon have always automatically the property that not only one common dissection is generated but even a whole family. If one shifts, e.g., the square tiling in Fig. 3.2 relative to the cross tiling then we get another common dissection, yet with less symmetry and possibly more pieces.

Common dissections that are generated by the tiling method have another property which reminds us of Observation 1.4: each edge of every piece of the dissection is in one of the two polygons on the outside and in the other on the inside, provided both tilings do not share common lines. (The example in Fig. 1.4 actually comes from a tiling of the plane by rectangles and triangles with common lines.) Thus we may take Observation 1.4 as an indication that the common dissection of altar and cube we found also comes from a tiling of space. We come back to this question later on.

Curiously the tiling method sometimes works even if one or both polygons do not allow a tiling of the plane. Let us try to dissect a genuine Swiss cross into a square: the leg of a Swiss cross is seven parts long but only six parts wide (this is constituted in a federal regulation: Bundesversammlung 1889). This cross only generates a tiling of the plane if one allowed to add little squares to fill the gaps between neighboring crosses, see Fig. 3.5. The point is that we can easily find a tiling of the plane using a square of the same area as the Swiss cross and the little square we introduced in the tiling with the cross. Also in this case we can match the periods of both tilings (see Fig. 3.6). We conclude that the Swiss cross and the square are equivalent by completion. In this case, the little square we introduced artificially does not bother! We have indeed a common dissection of the Swiss cross and a square with five pieces (Fig. 3.7). We observe that in the suitable orientation the four larger parts do not have to be rotated but only shifted from their place in the square to their place in the cross. Yet, the little square has to be rotated.

The world wide web is a source of lists of common dissections, see, e.g., Nowlen (2001) or Weisstein (2003). The book by Frederickson (1997) describes in addition numerous 


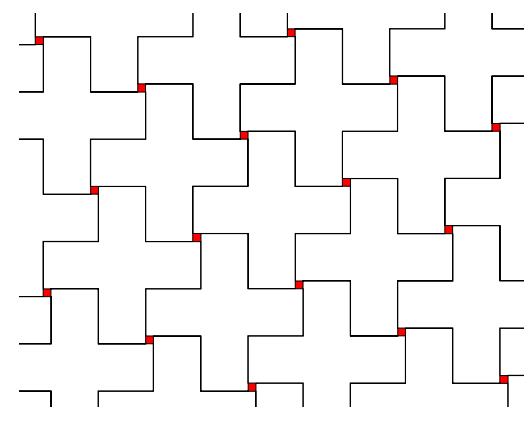

Fig. 3.5 Tiling by a Swiss cross

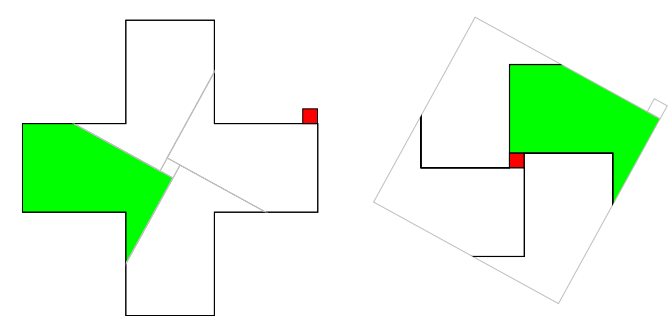

Fig. 3.7 Common dissection of the Swiss cross and a square

constructions and offers historical information. As an example we display three more dissections in Fig. 3.8. The dissection of the square and the octagon is obviously generated by an almost-tiling. Also the dissection of the dodecagon seems to have a similar origin but it does not come from a double tiling since several of the pieces are rotated, and there are some edges that are inside both figures (compare with the Swiss cross solution). For the dissection of the pentacle and the pentagon it is hard to tell how it was found but it does not look as if it originated from a double tiling...
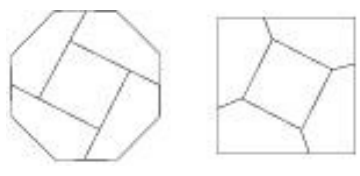
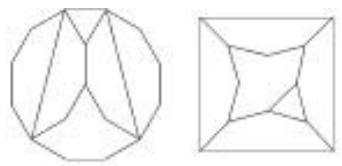

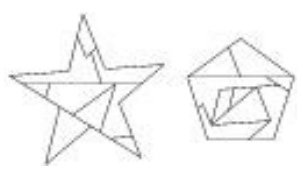

Fig. 3.8 More dissections

Also in space it is sometimes possible to find common dissections of polyhedra by the tiling method. Yet, this remains a limited tool: Hadwiger (1963) conjectured that, whenever a tiling of $\mathbb{R}^{d}$ is possible with congruent polyhedra, the polyhedron must be equidecomposable with a cube. This conjecture was proven by Groemer (1964), Hadwiger (1952b) and Mürner $(1975,1977)$ under different additional hypotheses concerning the kind of the tiling. Finally Debrunner (1980) was able to show that even the existence of a wellbalanced tiling implies that the Dehn invariant of the used polyhedron must be zero. (For 
a well-balanced tiling it is enough if every polyhedron used in the tiling is similar to the original polyhedron with a similarity constant bounded away from zero and infinity, and even finitely many polyhedral holes are allowed.) From Debrunner's theorem, it follows from the results of Sydler and Jessen that, in dimensions three and four, the polyhedron used for the tiling must then be equidecomposable to a cube. In particular, every quasicrystal, a solid which allows a non-periodic tiling of space and possibly even no periodic one, is equidecomposable to a cube.

Having now the tiling method at hand, we want to try to find the common dissection of the altar and the cube in seven pieces also by a double tiling of the space. To this end, we consider the cube

$$
\text { Cube }:=\left\{\sum_{i=1}^{3} x_{i} w_{i} \mid x_{i} \in[0,1]\right\},
$$

where $w_{i}=\sqrt[3]{2} e_{i}$ and the $e_{i}$ denote the three basis vectors in $\mathbb{R}^{3}$. For $i=1,2,3$ let $G_{\cdot i}$ be a (for the moment arbitrary) vector in $\mathbb{R}^{3}$ and $\Gamma=G \mathbb{Z}^{3}=\left\{\sum_{i=1}^{3} G_{\cdot i} \gamma_{i} \mid \gamma_{i} \in \mathbb{Z}\right\}$ the generated grid. Then $C u b e+\Gamma$ is a tiling of the space if the following conditions are satisfied:

$$
\begin{aligned}
G \cdot 1 & =w_{1}, \\
G \cdot 2 & =a w_{1}+w_{2}, \\
G \cdot 3 & =b w_{1}+c w_{2}+w_{3},
\end{aligned}
$$

where $a, b$ and $c$ are real numbers. Actually, up to a rotation and a translation, every tiling with a cube that is generated by a grid is of this form. The grid $\Gamma$ has the generating vectors

$$
G \cdot 1=\left[\begin{array}{c}
\sqrt[3]{2} \\
0 \\
0
\end{array}\right], \quad G \cdot 2=\left[\begin{array}{c}
\sqrt[3]{2} a \\
\sqrt[3]{2} \\
0
\end{array}\right], \quad G \cdot 3=\left[\begin{array}{c}
\sqrt[3]{2} b \\
\sqrt[3]{2} c \\
\sqrt[3]{2}
\end{array}\right]
$$

We choose $a_{1}^{\prime}=e_{1}, a_{2}^{\prime}=e_{2}$ and $a_{3}^{\prime}=2 e_{3}$, and consider the cuboid

$$
\text { Altar }^{\prime}:=\left\{\sum_{i=1}^{3} x_{i} a_{i}^{\prime} \mid x_{i} \in[0,1]\right\}
$$

which is spanned by these three vectors, and a grid $\Lambda=H \mathbb{Z}^{3}$. In order that Altar ${ }^{\prime}+\Lambda$ is a tiling, we aim to satisfy one of the following conditions:

$$
\begin{array}{ll}
\text { Variant 1: } & \text { Variant 2: } \\
H_{\cdot 1}=a_{1}^{\prime}, & H_{\cdot 1}=a_{3}^{\prime}, \\
H_{2}=r a_{1}^{\prime}+a_{2}^{\prime}, & H_{\cdot 2}=r a_{3}^{\prime}+a_{2}^{\prime}, \\
H \cdot 3=s a_{1}^{\prime}+t a_{2}^{\prime}+a_{3}^{\prime} . & H_{\cdot 3}=s a_{3}^{\prime}+t a_{2}^{\prime}+a_{1}^{\prime} .
\end{array}
$$

The grid $\Lambda$ has the following generating vectors in Variant 1 :

$$
H_{\cdot 1}=\left[\begin{array}{l}
1 \\
0 \\
0
\end{array}\right], \quad H_{\cdot 2}=\left[\begin{array}{l}
r \\
1 \\
0
\end{array}\right], \quad H_{\cdot 3}=\left[\begin{array}{l}
s \\
t \\
2
\end{array}\right],
$$


and in Variant 2:

$$
H_{\cdot 1}=\left[\begin{array}{l}
0 \\
0 \\
2
\end{array}\right], \quad H_{\cdot 2}=\left[\begin{array}{c}
0 \\
1 \\
2 r
\end{array}\right], \quad H_{\cdot 3}=\left[\begin{array}{c}
1 \\
t \\
2 s
\end{array}\right] .
$$

In order to have a common tiling of the space by the altar and the cube, the two grids $\Gamma$ and $\Lambda$ have to be congruent or mirror congruent. Now we observe at once that Variant 1 cannot lead to a common tiling: the shortest generating vector in $\Lambda$ has (for every choice of the parameters $r, s, t$ ) length 1 . On the other hand (for every choice of the parameters $a, b, c$ ) every non-zero vector in $\Gamma$ has at least length $\sqrt[3]{2}>1$. Thus we can restrict our attention to Variant 2. Since we are interested only in one particular solution, we try to find the parameters for which even both fundamental domains $G[0,1]^{3}$ and $H[0,1]^{3}$ are congruent or mirror congruent (and not only the grids). In order to have this it suffices that for a permutation $\sigma$ of the set $\{1,2,3\}$ the following is true:

$$
\left\langle G_{\cdot i} \mid G_{\cdot j}\right\rangle=\left\langle H_{\cdot \sigma(i)} \mid H_{\cdot \sigma(j)}\right\rangle
$$

for all $i \leq j \in\{1,2,3\}$. Now, it is easy to see that for $\sigma(1)=1$ there is no solution, since $\left\|G_{\cdot 1}\right\|=\sqrt[3]{2} \neq 2=\left\|H_{.1}\right\|$. It remains to consider four cases. From these, we retain only the one which actually leads to the solution, namely the cyclic shift $\sigma$ with $\sigma(1)=2$, $\sigma(2)=3, \sigma(3)=1$. In this case, the six equations are:

$$
\begin{aligned}
\sqrt[3]{4} & =1+4 r^{2}, \\
\sqrt[3]{4} a & =4 r s+t, \\
\sqrt[3]{4} b & =4 r \\
\sqrt[3]{4}\left(1+a^{2}\right) & =1+4 s^{2}+t^{2} \\
\sqrt[3]{4}(a b+c) & =4 s \\
\sqrt[3]{4}\left(1+b^{2}+c^{2}\right) & =4
\end{aligned}
$$

From (1), (3) and (6) it follows that

$$
\begin{aligned}
& r=-\frac{1}{2} \sqrt{\sqrt[3]{4}-1} \\
& b=-\sqrt[3]{2} \sqrt{\sqrt[3]{4}-1} \\
& c=-\sqrt{\sqrt[3]{4}-1}
\end{aligned}
$$

where we have chosen a sign for the root. The remaining three equations turn out not to be independent. Indeed, they can be solved for every choice of $a$. (The reason is that the volumes of the fundamental domains $G[0,1]^{3}$ and $H[0,1]^{3}$ agree by construction.) For $a=0$ we find $s=\frac{\sqrt[3]{4}}{4} \sqrt{\sqrt[3]{4}-1}$ and $t=\sqrt[3]{2}-\frac{1}{\sqrt[3]{2}}$ to solve the system. The resulting grid $\Gamma$ is therefore generated by the columns of the matrix

$$
G:=\left[\begin{array}{ccc}
\sqrt[3]{2} & 0 & -\sqrt[3]{4} \sqrt{\sqrt[3]{4}-1} \\
0 & \sqrt[3]{2} & -\sqrt[3]{2} \sqrt{\sqrt[3]{4}-1} \\
0 & 0 & \sqrt[3]{2}
\end{array}\right]
$$


This matrix is all you need to know to reconstruct the seven piece solution: an orthogonalization of the column vectors of $G$ in the natural order by the Gram-Schmidt method yields the spanning vectors of the cube: we find

$$
\begin{aligned}
& w_{1}=G \cdot 1, \\
& w_{2}=G \cdot 2-\frac{\left\langle w_{1} \mid G_{2}\right\rangle}{\left\langle w_{1} \mid w_{1}\right\rangle} w_{1}, \\
& w_{3}=G \cdot 3-\frac{\left\langle w_{1} \mid G \cdot 3\right\rangle}{\left\langle w_{1} \mid w_{1}\right\rangle} w_{1}-\frac{\left\langle w_{2} \mid G_{3}\right\rangle}{\left\langle w_{2} \mid w_{2}\right\rangle} w_{2},
\end{aligned}
$$

and get

$$
\begin{aligned}
\text { Cube } & =\sum_{i=1}^{3}[0,1] w_{i} \\
& \left.=\left\{\left[\begin{array}{ccc}
\sqrt[3]{2} & 0 & 0 \\
0 & \sqrt[3]{2} & 0 \\
0 & 0 & \sqrt[3]{2}
\end{array}\right] \mid \begin{array}{l}
a \\
b \\
c
\end{array}\right] \mid a, b, c \in[0,1]\right\} .
\end{aligned}
$$

If we shift this cube by elements of the grid we obtain the tiling of the space: $C u b e+G \mathbb{Z}^{3}$. Orthogonalization in the order given by $\sigma$, i.e., $\left(G_{.3}, G_{.1}, G_{.2}\right)$, yields the spanning vectors for the altar:

$$
a_{1}=G_{\cdot 3}, \quad a_{2}=G_{.1}-\frac{\left\langle a_{1} \mid G_{.1}\right\rangle}{\left\langle a_{1} \mid a_{1}\right\rangle} a_{1}, \quad a_{3}=G_{.2}-\frac{\left\langle a_{1} \mid G_{.2}\right\rangle}{\left\langle a_{1} \mid a_{1}\right\rangle} a_{1}-\frac{\left\langle a_{2} \mid G_{.2}\right\rangle}{\left\langle a_{2} \mid a_{2}\right\rangle} a_{2} .
$$

Hence

$$
\begin{aligned}
\text { Altar } & =\sum_{i=1}^{3}[0,1] a_{i} \\
& =\left\{\left[\begin{array}{ccc}
-\sqrt[3]{4} \sqrt{\sqrt[3]{4}-1} & \frac{\sqrt[3]{4}}{2} & 0 \\
-\sqrt[3]{2} \sqrt{\sqrt[3]{4}-1} & \frac{\sqrt[3]{2}}{2}-1 & \frac{\sqrt[3]{4}}{2} \\
\sqrt[3]{2} & \frac{\sqrt[3]{2} \sqrt{\sqrt[3]{4}-1}}{2} & \frac{\sqrt[3]{4} \sqrt{\sqrt[3]{4}-1}}{2}
\end{array}\right]\left[\begin{array}{l}
a \\
b \\
c
\end{array}\right] \mid a, b, c \in[0,1]\right\} .
\end{aligned}
$$

If we shift this altar by elements of the grid we obtain the tiling of the space: Altar $+G \mathbb{Z}^{3}$.

Now, the altar intersects precisely seven of the shifted cubes (and vice versa). And the intersection sets are just the seven pieces we found earlier! By construction, they build the altar. But since both tilings share the same grid, they can be reassembled to build the cube. In Fig. 3.9 we can see how the altar lies in the seven cubes. The arrows indicate the basis of the grid $G_{.1}, G_{.2}, G_{.3}$; two of them point into directions of edges of the cube, the third one in the direction of the hidden long edge of the altar.

Further investigations lend some support to the conjecture that the solution in seven pieces which we found is minimal among all solutions which are generated by the tiling method. 


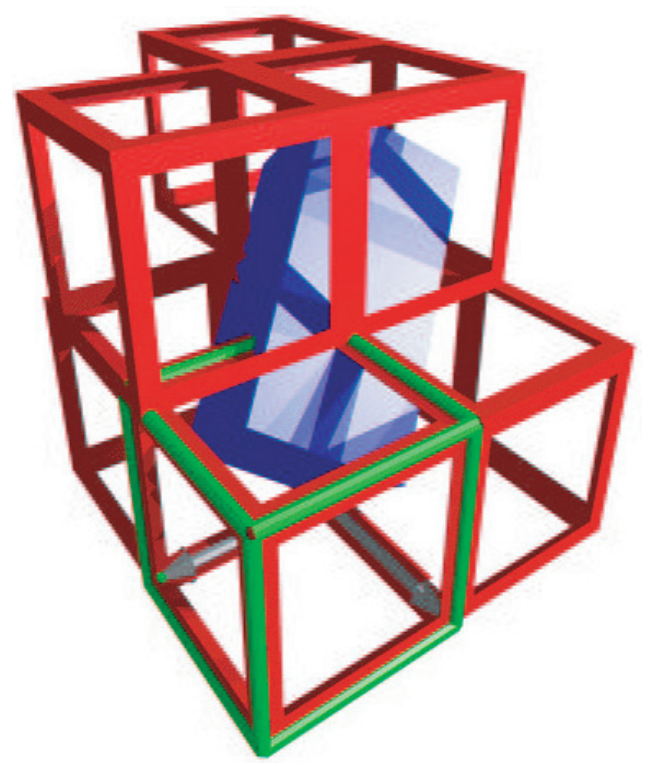

Fig. 3.9 Seven pieces by double tiling
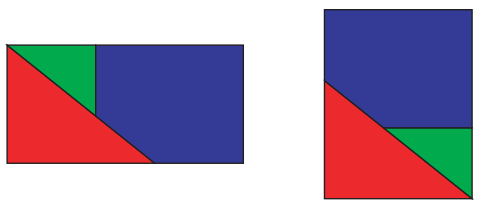

Fig. 3.10 A P-slide

Concerning solutions without any restriction, "in the very last pocket", we discovered that Specker's question had already been posed over 70 years ago by Cheney (1932). An eight piece solution by Ransom was published in Ransom (1933), and it is mentioned that the proposer solved the problem as well. This eight piece solution is similar to the one we found first. Cheney also indicates that, since that solution was sent to the printer, he had learned of a seven piece solution by Wheeler. This solution was published in Wheeler (1935). It was based on a different rectangle metamorphosis that puzzlers call the P-slide (see Fig. 3.10). This P-slide is not induced by a proper double tiling, since the two involved translations are parallel. As a consequence, Wheeler's seven piece solution is not induced by a double tiling but still needs only translations. However, it does not have the inside out property of our seven piece solution. Later on, Goldberg (1966) published a duplication of the cube by dissection and a hinged linkage: The ten pieces of his solution can be hinged in such a way, that the pieces roll over from the altar position to the cube. Then, half a century after Cheney proposed the problem, Hanegraaf (1989) improved the solution again and reduced, by using two P-slides, the number of pieces to six. The key is to avoid intersection of the first and second stage cuts: 

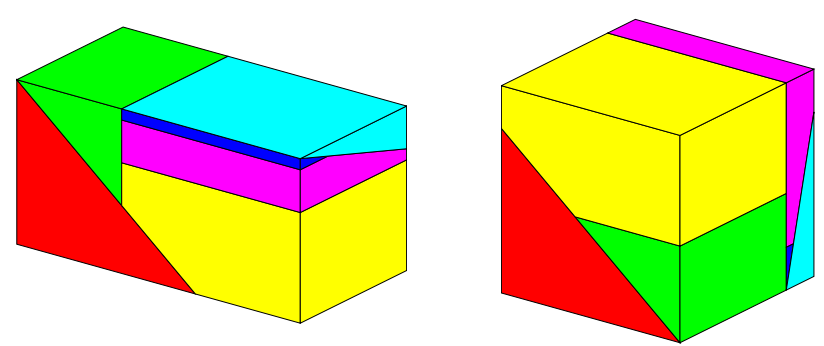

Fig. 3.11 Hanegraaf's six piece solution

Theorem 3.1 (Hanegraaf (1989)). The metamorphosis from altar to cube can be done with six pieces.

However, the six piece solution needs rotation of some pieces and an inside out property like Observation 1.4 is not valid any more. To our knowledge, Hanegraaf's solution is the minimal known today. Yet, though it seems optimal, puzzlers seemingly never tried or succeeded in proving this or similar optimality results and the lower bound question remains open.

Open Question 3.2. Is it possible to achieve a six piece solution

- using the tiling method?

- with the inside out property?

- with translations only?

Is it possible to achieve a solution with five pieces?

We conjecture that Theorem 3.1 is optimal in the general setting and Theorem 1.3 is optimal when only translations are allowed or the inside out property is required.

\section{Acknowledgements}

We would like to thank our colleagues who had to stand our sometimes overwhelming enthusiasm. In particular, we would like to thank Douglas G. Rogers for his remarks. And, last but not least, our gratitude goes to Ernst Specker who raised this wonderful problem.

\section{References}

Boltîanksĭ̌, Vladimir G. (1978). Hilbert's Third Problem. Scripta Series in Mathematics. John Wiley \& Sons, New York, Toronto, London, Sydney. Translation of Tret'ia problema Gil'berta.

BOLYAI, FARKAS (1832). Tentamen iuventutem studiosam in elementa matheseos purae dementaris ac sublimioris methodo intuitiva evidentiaque huic propria introducendi, cum appendice triplici. Acad. scient. hungar., Budapestini, 2nd edition.

BUNDESVERS AMMLUNG DER SCHWEIZERISCHEN EIDGENOSSENSCHAFT (1889). Bundesbeschluss betreffend das eidgenössische Wappen, volume 1 of Bereinigte Sammlung der Bundesgesetze und Verordnungen 1848-1947, 147. Schweizerische Bundeskanzlei 1949-1955, Bern.

Cheney, JR., William Fitch (1932). Problem E4. Amer. Math. Monthly 39 (1932), 489. 
Debrunner, Hans E. (1980). Über Zerlegungsgleichheit von Pflasterpolyedern mit Würfeln. Arch. Math. 35 (1980), 583-587.

DEHN, MAX (1900). Über raumgleiche Polyeder. Nachrichten von der Königlichen Gesellschaft der Wissenschaften zu Göttingen, Mathematisch-physikalische Klasse (1900), 345-354.

URL http : / / www-gdz . sub . uni-goettingen. de/.

DeHn, MAX (1900). Über den Rauminhalt. Math. Ann. 55 (1900), 465-478.

URL http: / / www-gdz . sub. uni-goettingen. de/.

This is the very same article that Hilbert (1900) cites as „Raumteilungen“.

Dehn, MaX (1902). Über Zerlegung von Rechtecken in Rechtecke. Math. Ann. 57 (1902), 314-332.

URL http : / / www-gdz . sub . uni-goettingen. de/.

DUPONT, JOHAN L. (2001). Scissors congruences, group homology and characteristic classes. Number 1 in Nankai Tracts in Mathematics. Nankai Institute of Mathematics.

URL http: //home.imf .au.dk/dupont/scissors.ps.

Dupont, JOHAN L. \& SAH, CHIH-HAN (1990). Homology of Euclidean groups of motions made discrete and Euclidean scissors congruence. Acta Math. 164 (1990), 1-27.

Frederickson, Greg Norman (1997). Dissections: Plane \& fancy. Cambridge University Press.

Gauss, Carl Friedrich (1844). Congruenz und Symmetrie, volume 8 of Werke, 241, 244. B.G. Teubner, 1900, Leipzig. Zwei Briefe an Gerling, 8. und 17. April 1844.

GERWIEN, P. (1833). Zerschneidung jeder beliebigen Anzahl von gleichen geradlinigen Figuren in dieselben Stücke. J. Reine Angew. Math. 10 (1833), 228-234.

Goldberg, Michael (1966). A duplication of the cube by dissection and a hinged linkage. Mathematical Gazette 50 (1966), 304-305.

Groemer, Helmut (1964). Über Würfel- und Raumzerlegungen. Elem. Math. XIX(2) (1964), 25-27.

HAdwiger, Hugo (1952a). Ergänzungsgleichheit k-dimensionaler Polyeder. Math. Z. 55(3) (1952), $292-298$.

HADWIGER, HUGO (1952b). Mittelpunktspolyeder und translative Zerlegungsgleichheit. Math. Nachr. 8 (1952), 53-58.

HAdWiger, Hugo (1952c). Über addierbare Intervallfunktionale. Tohoku Math. J. 4(2) (1952), 33-37.

HADWIGER, Hugo (1957). Vorlesungen über Inhalt, Oberfläche und Isoperimetrie. Number XCIII in: Grundlehren der mathematischen Wissenschaften. Springer-Verlag, Berlin, Göttingen, Heidelberg.

Hadwiger, Hugo (1963). Ungelöste Probleme, Nr. 45. Elem. Math. 18 (1963), 29-31.

Hadwiger, Hugo \& Glur, Paul (1951). Zerlegungsgleichheit ebener Polygone. Elem. Math. 6(5) (1951), 97-106.

HAnegraAf, Anton (1989). The Delian altar dissection. Self-published, Elst, the Netherlands. First booklet in his projected series Polyhedral Dissections.

HeAth, Thomas L. (ed.) (1925). The thirteen books of Euclid's elements, volume 1. Dover Publications, Inc., New York, Second edition. First edition appeared 1908. Translated from the text of Heiberg.

Hilbert, DAVID (1900). Mathematische Probleme. Nachrichten von der Königlichen Gesellschaft der Wissenschaften zu Göttingen (1900), 253-297. URL http : / / www-gdz . sub . uni-goettingen. de/ Archiv für Mathematik und Physik, 3. Reihe 1 (1901), 44-63 and 213-237. English translation: Mathematical Problems, Bulletin of the American Mathematical Society 8 (1902), 437-479.

Hill, Micaiah John Muller (1896). Determination of the Volumes of Certain Species of Tetrahedra Without Employment of the Method of Limits. Proc. London Math. Soc. 27 (1896), 39-52.

JEsSEn, BøRGE (1968). The algebra of polyhedra and the Dehn-Sydler theorem. Math. Scand. 22 (1968), 241-256.

Jessen, Børge (1972). Zur Algebra der Polytope. Nachr. Akad. Wiss. Göttingen Math.-Phys. Kl. II (1972), 47-53.

Kellerhals, RUth (1999). Old and new about Hilbert's third problem. In European women in mathematics, Proceedings of the 9th General Meeting (Loccum, 1999), CAMINA, RACHEL \& FAJSTRUP, LiSBETH, eds., 179187. Hindawi Publishing Corporation.

URL http : / / www . hindawi.dk/books/977594502X/B977594502X000278.pdf. 
LOWRY (1814). Solution to question 269, [proposed] by Mr. W. Wallace. In New series of the Mathematical Repository, LEYBOURN, T., ed., volume III, part I, 44-46. W. Glendinning, London.

MacMahon, Percy A. (1922). Pythagoras's Theorem as a Repeating Pattern. Nature 109(2737) (1922), 479. Mahlo, PAUl (1908). Topologische Untersuchugen über Zerlegung in ebene und sphärische Polygone. Inaugural-Dissertation, Vereinigte Friedrichs-Universität Halle-Wittenberg, Halle a.S., Germany.

Mürner, Peter (1975). Translative Parkettierungspolyeder und Zerlegungsgleichheit. Elem. Math. 30(2) (1975), 25-27.

MÜRner, PETER (1977). Untersuchungen auf dem Gebiet der Zerlegungsgleichheit Euklidischer Polytope bezüglich ausgewählter Bewegungsgruppen und über entsprechende Parkettierungen des d-dimensionalen Raumes. Inaugural-Dissertation, Universität Bern.

NOWLEN, CATHY (2001). History of equidecomposability. Webpage.

URL http: / / www.geocities. com/cnowlen/Cathy/Math5200/equidecom.html.

NÜSKEN, MiChAEL (2003). Delian Metamorphoses (Delische Verwandlungen). Webpage.

URL http: / / www . math. uni-paderborn. de/ nuesken/wrt/delos/.

Ransom, W.R. (1933). Problem E4, Solution. The American Mathematical Monthly 40 (1933), 113-114. Amer. Math. Monthly 40 (1933), 113-114. ISSN 0002-9890.

Rogers, Douglas G. (2005). Pythagoras drawn, quartered by Perigal and Thâbit. In preparation (2005).

SCHÖBI, PHILliPP (1985). Ein elementarer und konstruktiver Beweis für die Zerlegungsgleichheit der Hill'schen Tetraeder mit einem Quader. Elem. Math. 40 (1985), 85-97.

SPANDAW, JEROEN (2002). Dissecting Cuboids into Cuboids.

URL http: //www-ifm. math. uni-hannover. de/html/preprints.html.

Preprint pr307, Institut für Mathematik, Universität Hannover.

SPECKER, ERnSt (2002). Aufgabe 1184. Elem. Math. 57(3) (2002), 133.

URL http: / / ink. springer.de/link/service/journals/00017/tocs/t2057003.htm.

SydLER, JEAN-PIERRE (1943/44). Sur la décomposition des polyèdres. Comment. Math. Helv. 16 (1943/44), 266-273.

SYDLER, JEAN-PIERRE (1965). Conditions nécessaires et suffisantes pour l'équivalence des polyèdres de l'espace euclidien à trois dimensions. Comment. Math. Helv. 40 (1965), 43-80.

Thaer, Clemens (ed.) (1937). Die Elemente von Euklid, V. Teil (Buch XI-XIII). Number 243 in Ostwald's Klassiker der exakten Wissenschaften. Akademische Verlagsgesellschaft m.b.H., Leipzig. Nach Heibergs Text aus dem Griechischen übersetzt.

Wallace, William (ed.) (1831). Elements of geometry. Bell \& Bradfute, Edinburgh, 8th edition. First six books of Euclid, with a supplement by John Playfair.

WEISSTEIN, ERIC W. (2003). Dissection. Webpage, part of Eric Weisstein's world of Mathematics.

URL http: / / mathworld. wolfram. com/Dissection. html.

Wheeler, Albert H. (1935). Problem E4, Solution. Amer. Math. Monthly 42 (1935), 509-510.

Norbert Hungerbühler

Departement für Mathematik

Universität Freiburg, Pérolles

CH-1700 Freiburg, Schweiz

e-mail: norbert.hungerbuehler@unifr.ch

http: //www . unifr.ch/perso/hungerbu/

Michael Nüsken

b-it (Bonn-Aachen International Center

for Information Technology)

Görresstr. 13

D-53113 Bonn, Deutschland

e-mail: nuesken@bit.uni-bonn.de

http: //www . math. upb. de/ nuesken/ 